\title{
Abdominal wall mass suspected of endometriosis: clinical and pathologic features
}

\author{
HeeKyoung Song, MD, SangHee Lee, MD, Min Jeong Kim, MD, PhD, Jae Eun Shin, MD, PhD, \\ Dae Woo Lee, MD, PhD, Hae Nam Lee, MD, PhD \\ Department of Obstetrics and Gynecology, Bucheon St. Mary's Hospital, College of Medicine, The Catholic University of Korea, Seoul, Korea
}

\section{Objective}

To evaluate the clinical and pathological characteristics of lower anterior abdominal wall masses suspicious for endometriosis.

\section{Methods}

A retrospective review of 38 patients who underwent surgery for a lower anterior abdominal wall mass suspicious for endometriosis was performed. Those with skin and intraperitoneal masses, lipomas, hernias, and metastatic malignant masses were excluded. Patient age, body mass index, delivery history, dysmenorrhea, and mass size and location were analyzed.

\section{Results}

Thirty-seven $(97.3 \%)$ patients had a relevant surgical history, including $35(92.1 \%)$ with a history of cesarean section $(C / S)$. Among the three patients with no history of $C / S, 1$ underwent total abdominal and another total laparoscopic hysterectomy, and 1 had no previous surgical history. The mean ( \pm standard deviation) size of the abdominal masses was $3.2 \pm 1.2 \mathrm{~cm}$. One patient developed a recurrent mass after excision of abdominal wall endometriosis. Trocar site endometrioma was found in one patient following total laparoscopic hysterectomy. According to the final pathology reports, endometriosis was found in $35(92.1 \%)$ of patients. The remaining 3 patients $(7.9 \%)$ had malignancy: adenocarcinoma, squamous cell carcinoma, and extra-gastrointestinal stromal tumor. Before surgery, only 3 patients (7.9\%) underwent fine-needle aspiration biopsy of the masses, which were all postoperatively confirmed to be pathologically benign.

\section{Conclusion}

Although most abdominal wall masses in the present sample were endometriosis occurring at the scar site from a previous operation, $7.9 \%$ of patients ultimately exhibited malignancy. Therefore, all patients with suspected anterior wall endometriosis should undergo preoperative biopsy to identify the few that will have an alternative diagnosis.

Keywords: Endometriosis

\section{Introduction}

Endometriosis is characterized by the presence of stroma and endometrial glands outside the uterine cavity. This condition affects approximately $15 \%$ to $40 \%$ of women of childbearing age. The most common site of endometriosis is the abdominal cavity, specifically the pelvis, and occasionally, extra-pelvic sites [1]. Abdominal wall endometriosis (AWE) is a rare condition characterized by the presence of endometrial tissue in the subcutaneous fatty layer or muscles of the abdominal wall. AWE is usually caused by the dissemination
Received: 2019.05.27. Revised: 2019.10.16. Accepted: 2019.11.06. Corresponding author: Hae Nam Lee, MD, PhD

Department of Obstetrics and Gynecology, Bucheon St. Mary's Hospital, College of Medicine, The Catholic University of Korea, 327 Sosa-ro, Bucheon 14647, Korea

E-mail: leehn@catholic.ac.kr

https://orcid.org/0000-0002-8861-508X

Articles published in Obstet Gynecol Sci are open-access, distributed under the terms of the Creative Commons Attribution Non-Commercial License (http://creativecommons. org/licenses/by-nc/3.0/) which permits unrestricted non-commercial use, distribution, and reproduction in any medium, provided the original work is properly cited.

Copyright $\odot 2020$ Korean Society of Obstetrics and Gynecology 


\title{
Obstetrics \& Gynecology Science
}

\author{
Vol. 63, No. 3, 2020
}

of endometrial tissue at the incision site during obstetrical or gynecological surgery $[2,3]$. Malignant transformation of AWE is extremely rare. The most common malignant pathologies, reported in several studies, are clear cell or endometrioid carcinomas [1,4-6]. Endometriosis-associated abdominal wall cancers exhibit aggressive prognoses [4]. However, the estimated prevalence of AWE, especially malignant transformation of AWE, is no higher than $1 \%$. Therefore, few investigations have examined AWE and malignant transformation, and most reports have been case studies or literature reviews $[5,7,8]$.

In the present study, patients with clinically presumed AWE were evaluated. Pathological analyses of the abdominal wall masses made it possible to identify deficiencies in clinical procedures and make recommendations to improve management and prognosis.

\section{Materials and methods}

Eligible patients, seen in Bucheon, Seoul, Incheon, Uijeongbu at St. Mary's Hospital, St. Vincent Hospital, or the College of Medicine at The Catholic University of Korea (Seoul, Korea), were identified based on surgical history of suspected endometriosis associated with a lower anterior abdominal wall mass. Medical records between January 2010 and November 2017 were evaluated with regard to patient age, mass size and location, symptoms, and final permanent pathology following surgical resection. In all cases, the abdominal mass was situated between the umbilicus and symphysis pubis of the lower anterior abdominal wall. The inclusion criterion was a lower anterior abdominal wall mass suspicious for endometriosis before surgery. Patients with skin masses, intraperitoneal masses, lipomas, and hernias were excluded. Masses originating from a known malignancy (5 patients) were also excluded. Age, body mass index, delivery method and number, dysmenorrhea, menorrhagia, symptoms, mass size and location, and permanent pathology were analyzed. Cyclic pain of the abdominal mass, apart from previous dysmenorrhea, was analyzed separately. It was not possible to collect patient history of menorrhagia or dysmenorrhea among those who had previously undergone total hysterectomy. Abdominal wall mass size was reported according to long axis $(\mathrm{cm})$.

\section{Results}

The mean ( \pm standard deviation) patient age was $38.9 \pm 6.7$ years, and the mean abdominal mass size was $3.2 \pm 1.2 \mathrm{~cm}$ (Table 1). Seventeen patients (44.7\%) had dysmenorrhea, and $4(10.5 \%)$ experienced pain from the abdominal mass during menstruation. Of 31 patients with available medical records, the interval from the most recent previous surgery to abdominal wall mass diagnosis was $5.93 \pm 4.29$ years. Although several patients complained of dysmenorrhea or menorrhagia before the latest surgery, endometriosis or endometrial cyst had not been confirmed at the previous surgeries. After discovery of the abdominal wall mass, 1 patient received gonadotrophin-releasing hormone agonist, and another took oral contraceptives to control pain and reduce the size of the mass. These treatments were ineffective; therefore, both patients ultimately underwent surgery. An ovarian endometrial cyst was found in 1 patient on magnetic resonance imaging (MRI) performed to evaluate the abdominal wall mass; this patient underwent laparoscopic ovarian cystectomy and abdominal wall mass removal.

Thirty-seven of the 38 patients (97.3\%) had a history of surgery, including 35 (92.1\%) who previously underwent cesarean section (C/S). Among the 3 patients with no history of $\mathrm{C} / \mathrm{S}, 1$ underwent total abdominal and another total laparo-

Table 1. Characteristics of patients with abdominal wall mass $(n=38)$

\begin{tabular}{lc}
\hline Characteristics & \multicolumn{1}{c}{ Values } \\
\hline Mean age $(y r)$ & $38.9 \pm 6.7(36.6-41.1)$ \\
Mean BMI & $22.9 \pm 4(21.7-24.2)$ \\
Mean delivery number & $1.6 \pm 0.7(1.4-1.8)$ \\
Delivery mode & $35(92.1)$ \\
Cesarean section delivery & $3(7.9)$ \\
Vaginal delivery & $37(97.3)$ \\
History of surgery & $35(92.1)$ \\
Cesarean section & $1(2.6)$ \\
Total hysterectomy & $1(2.6)$ \\
Total laparoscopic hysterectomy & $10(26.3)$ \\
Menorrhagia & $17(44.7)$ \\
Dysmenorrhea & \\
\hline
\end{tabular}

Values are presented as mean \pm standard deviation (95\% confidence interval) or number (\%).

$\mathrm{BMI}$, body mass index. 


\title{
Obstetrics \& Gynecology Science
}

\author{
HeeKyoung Song, et al. Abdominal wall endometriosis
}

scopic hysterectomy, and 1 had no previous related/relevant surgical history. All 37 patients with a relevant surgical history exhibited a mass at the surgical scar.

The final pathology reports described endometriosis in 35 of the 38 patients (92.1\%). Trocar site endometrioma was found in 1 patient with previous total laparoscopic hysterectomy. One (2.6\%) patient experienced a recurrent mass 6 years after previous AWE excision $(10 \mathrm{~cm})$ that was larger than the initial mass $(5 \mathrm{~cm})$. A mesh was inserted during surgery for the recurrent mass. Due to incomplete medical records, it was not possible to collect information regarding resection margins from the pathology and surgical reports for all cases. Among the 35 patients, 25 (71.4\%) did not attend outpatient clinic follow-up for $>1$ year.

Three of the 38 patients (7.9\%) were diagnosed with malignancy: adenocarcinoma-originated endometrium or endocervix, squamous cell carcinoma, and extra-gastrointestinal stromal tumor (EGIST) (Table 2). The patient diagnosed with adenocarcinoma of the mass, who underwent a previous $C / S$,

Table 2. Characteristics of abdominal wall mass $(n=38)$

\begin{tabular}{lc}
\hline Characteristics & Values \\
\hline Presenting symptom & \\
Pain on abdominal mass not related to menstruation ${ }^{\text {a) }}$ & $1(2.6)$ \\
Pain on abdominal mass related to menstruation ${ }^{b}$ & $4(10.5)$ \\
Palpable mass ${ }^{c}$ & $25(65.8)$ \\
a) + c) & $1(2.6)$ \\
b) + c) & $7(18.4)$ \\
Mass location & \\
Midline of abdomen & $10(26.3)$ \\
Right side & $12(31.6)$ \\
Left side & $14(36.8)$ \\
Bilateral & $2(5.3)$ \\
Subcutaneous & $9(23.7)$ \\
Subfascia & $14(36.8)$ \\
Muscle & $15(39.5)$ \\
Located in Cesarean section scar & $35(92.1)$ \\
Not located in Cesarean section scar & $2(5.3)$ \\
Pathology & $1(2.6)$ \\
Endometriosis & \\
Squamous cell carcinoma & \\
Adenocarcinoma & $15(92.1)$ \\
\hline Values are presented as number (\%). & $1(2.6)$ \\
\hline
\end{tabular}

Values are presented as number (\%). was confirmed to have malignant transformation of the endometrium or endocervix. Her immunohistochemical results were $\mathrm{ER}(+)$, PAX8(+), GATA3(-), and WT-1(-). The patient diagnosed with squamous cell carcinoma had previously undergone total abdominal hysterectomy due to carcinoma in situ 20 years previously. The patient with no previous surgical history was pathologically confirmed with EGIST of the abdominal mass.

Thirty-seven patients underwent diagnostic imaging studies using computed tomography (CT) or MRI before abdominal surgery; however, no suspicious findings suggesting malignancy were observed on any scan. Before surgery, 3 patients (7.9\%) underwent fine-needle aspiration biopsy (FNAB) of the abdominal wall mass, all of which were postoperatively confirmed to be pathologically benign. None of the patients with malignancy underwent presurgical biopsy.

\section{Discussion}

AWE accounts for $0.4 \%$ to $2.0 \%$ of patients diagnosed with endometriosis. It is found predominantly in the umbilical scar and scars from previous abdominal incisions, especially $C / S$, laparoscopy, or appendectomy [1]. In the present study, 34 of 35 patients with definitive AWE had a history of $C / S$; the other had undergone laparoscopic surgery. Their abdominal wall masses were situated at the scars associated with $\mathrm{C} / \mathrm{S}$ or laparoscopic trocar sites. Several studies have reported port site endometriomas after laparoscopic surgery. Emre et al. [9] reported 15 cases and Siddiqui et al. [10] reported a case study of port site endometrioma. In the present study, the patient who had not undergone surgery had a final pathology report of EGIST. These data suggest that surgeons should consider the possibility of scar site endometrioma when operating. Ozel et al. [11] suggested that some C/S practices, such as certain bleeding control methods, abdominal cavity washing before closure, and minimizing subcutaneous dead space, may reduce the incidence of AWE. Surgical excision should include clear margins, at least $1 \mathrm{~cm}$ from solid tissue. In addition, medications such as gonadotrophinreleasing hormone agonist or danazol (a synthetic derivative of 17-a-ethinyl testosterone), both before and after surgical resection, may be effective in preventing AWE [12-14].

Malignant transformations of AWE are extremely rare, with clear cell carcinoma and adenocarcinomas reported in the 


\section{Obstetrics \& Gynecology Science}

Vol. 63, No. 3, 2020

literature [4-7]. Taburiaux et al. [4] reported that 26 cases of malignant transformation of AWE were published between 1986 and 2014, among which the most common histological type was clear cell carcinoma, reported in 17 patients (65\%). Other histological types in that report included endometrioid carcinoma in 6 patients (23\%), serous papillary carcinoma in $2(8 \%)$, and sarcoma in $1(4 \%)$. Furthermore, de Almeida Gentile et al. [1] noted that malignant transformation involving the abdominal wall is extremely rare, with fewer than 30 cases of endometrioid carcinoma (70\%), sarcoma (25\%), and clear cell carcinoma (5\%) reported worldwide. In the present study, 3 malignant abdominal masses were identified as adenocarcinoma of endometrial or endocervical origin, metastatic abdominal mass derived from cervical cancer, and EGIST, respectively. Most abdominal wall masses in females are benign; therefore, the malignancy rate reported in the present article is remarkable. Precancerous carcinoma in situ seldom recurs or metastasizes after total hysterectomy, especially as metastasis to the distant abdominal wall as cancer after a significant delay; therefore, we did not expect abdominal wall squamous cell carcinoma metastasis. Abdominal wall EGIST is also rare, with only a few cases reported $[15,16]$. After diagnosis of EGIST in the abdominal wall, we transferred this patient to the general surgery department.

In the present study, only 3 patients underwent FNAB before surgical treatment. In the study by Bektaş et al. [17], preoperative diagnosis of a lower anterior abdominal wall mass suspicious for endometriosis included $47.5 \%$ endometriosis, 20\% abdominal wall tumor, 25\% hernia, 5\% desmoid tumor, and $2.5 \%$ suture granuloma. The abdominal wall mass was likely a tumor, although only $20 \%$ of patients underwent FNAB. Endometriosis was present in only $50 \%$ of those with a mass described in the final pathology report.

We analyzed the medical records of 28 of 35 patients who had not undergone FNAB, excluding 7 with incomplete medical records. The mean interval from original surgery to presentation of symptoms associated with the suspected AWE involving the abdominal wall mass was 14.1 months (range, 1-72 months). This delay in the treatment decision was due not only to delayed clinical visits by the patients, but also delayed decision-making by surgeons who overlooked possible malignancy. In the study by Horton et al. [18], this interval was 3.6 years.

According to several published studies, the incidence of AWE is low and mimics other clinical conditions, making pre- operative diagnosis more difficult. Ultrasound, CT, and MRI are used to diagnose AWE $[12,19]$; however, these studies are not useful because they do not report significant findings. On ultrasound, AWE appears as a solid, heterogeneous hypoechoic mass with inner echogenic spots. The echogenic patterns depend on the hemorrhagic and fibrous components of the lesions. Although attenuation may vary, mild-tomoderate enhancement of the lesion has been observed on $\mathrm{CT}$ scans of the abdominal wall close to the $\mathrm{C} / \mathrm{S}$ incision site [20]. In the present study, we did not detect any malignant masses on preoperative imaging. Therefore, we recommend FNAB for any abdominal mass suspicious for endometriosis before deciding on a treatment plan. According to Medeiros et al. [21], FNAB is a fast and accurate preoperative diagnostic method that prevents errors in approaching AWE. A history of confirmed pathological diagnoses may also improve patient prognosis. However, the use of FNAB is controversial due to the risk for new implants at puncture sites [12,22]. To avoid the spread of endometriosis after these procedures, it is advisable to include the aspiration site in the surgical resection field. Before performing FNAB, abdominal hernia should always be ruled out $[23,24]$.

Several studies have reported the rare recurrence of AWE. Bektaş et al. [17] reported a higher rate of recurrence (9.1\%) than in previous inadequate resection(s) of endometriotic lesions; another study reported a rate of $7.5 \%$, and 1 review indicated a recurrence rate of 4.5\% [24]. Many reports suggest that adequate surgical excision $(1 \mathrm{~cm})$ is essential for favorable outcomes [13]; however, to our knowledge, no studies have addressed the role of surgical margin size in recurrence. Although we have reported a patient with recurrent AWE, further studies are needed.

The present study was limited by the selection bias inherent to all retrospective designs, although this remains the best approach to investigating rare diseases such as AWE. Because we analyzed only 38 patients, statistical power may have been low. The major strength of the present study was that patients from 5 hospitals were included, increasing the generalizability of the results.

In conclusion, we found that most abdominal wall masses in patients whose histories we reviewed were benign endometrioses, and that all AWEs occurred at previous C/S sites. However, $7.9 \%$ of patients with a lower anterior abdominal wall mass suspicious for endometriosis turned out to have malignancy. Therefore, all patients with suspected AWE 


\section{Obstetrics \& Gynecology Science}

HeeKyoung Song, et al. Abdominal wall endometriosis

should undergo preoperative biopsy to identify the few that will have an alternative diagnosis.

\section{Conflict of interest}

No potential conflict of interest relevant to this article was reported.

\section{Ethical approval}

This study was approved by the Institutional Review Board (IRB No.: HC 19RESI0011) of St. Mary's Hospital.

\section{Patient consent}

This study approved the patient consent waiver due to the retrospective study design.

\section{References}

1. de Almeida Gentile J, Migliore R, Kistenmacker FJN, Oliveira MM, Garcia RB, Bin FC, et al. Malignant transformation of abdominal wall endometriosis to clear cell carcinoma: case report. Sao Paulo Med J 2018;136:58690.

2. Rindos NB, Mansuria S. Diagnosis and management of abdominal wall endometriosis: a systematic review and clinical recommendations. Obstet Gynecol Surv 2017;72:116-22.

3. Blanco RG, Parithivel VS, Shah AK, Gumbs MA, Schein M, Gerst PH. Abdominal wall endometriomas. Am J Surg 2003;185:596-8.

4. Taburiaux L, Pluchino N, Petignat P, Wenger JM. Endometriosis-associated abdominal wall cancer: a poor prognosis? Int J Gynecol Cancer 2015;25:1633-8.

5. Shalin SC, Haws AL, Carter DG, Zarrin-Khameh N. Clear cell adenocarcinoma arising from endometriosis in abdominal wall cesarean section scar: a case report and review of the literature. J Cutan Pathol 2012;39:103541.

6. Modesitt SC, Tortolero-Luna G, Robinson JB, Gershenson
DM, Wolf JK. Ovarian and extraovarian endometriosisassociated cancer. Obstet Gynecol 2002;100:788-95.

7. Ferrandina G, Palluzzi E, Fanfani F, Gentileschi S, Valentini AL, Mattoli MV, et al. Endometriosis-associated clear cell carcinoma arising in caesarean section scar: a case report and review of the literature. World J Surg Oncol 2016;14:300.

8. Van Gorp T, Amant F, Neven P, Vergote I, Moerman P. Endometriosis and the development of malignant tumours of the pelvis. A review of literature. Best Pract Res Clin Obstet Gynaecol 2004;18:349-71.

9. Emre A, Akbulut S, Yilmaz M, Bozdag Z. Laparoscopic trocar port site endometriosis: a case report and brief literature review. Int Surg 2012;97:135-9.

10. Siddiqui ZA, Husain F, Siddiqui Z, Siddiqui M. Port site endometrioma: a rare cause of abdominal wall pain following laparoscopic surgery. BMJ Case Rep 2017;2017:bcr-2017-219291.

11. Ozel L, Sagiroglu J, Unal A, Unal E, Gunes P, Baskent E, et al. Abdominal wall endometriosis in the cesarean section surgical scar: a potential diagnostic pitfall. J Obstet Gynaecol Res 2012;38:526-30.

12. Patterson GK, Winburn GB. Abdominal wall endometriomas: report of eight cases. Am Surg 1999;65:36-9.

13. Kim SM, Kim HK, Namkung J, Song JY, Jung IC, Cho $\mathrm{HH}$, et al. A case of reconstruction of abdominal fascia using polypropylene mesh for patient of abdominal wall endometriosis after cesarean section. Korean J Obstet Gynecol 2012;55:517-21.

14. Purvis RS, Tyring SK. Cutaneous and subcutaneous endometriosis. Surgical and hormonal therapy. J Dermatol Surg Oncol 1994;20:693-5.

15. Alkhatib L, Albtoush O, Bataineh N, Gharaibeh K, Matalka I, Tokuda Y. Extragastrointestinal Stromal Tumor (EGIST) in the abdominal wall: case report and literature review. Int J Surg Case Rep 2011;2:253-5.

16. He $X$, Chen N, Lin L, Wang C, Wang Y. Extragastrointestinal stromal tumor of the abdominal subcutaneous tissue: report of a very rare case at an unusual site. J Int Med Res 2017;45:1273-8.

17. Bektaş H, Bilsel Y, Sari YS, Ersöz F, Koç O, Deniz M, et al. Abdominal wall endometrioma; a 10-year experience and brief review of the literature. J Surg Res 2010;164:e77-81.

18. Horton JD, Dezee KJ, Ahnfeldt EP, Wagner M. Abdomi- 


\section{Obstetrics \& Gynecology Science}

Vol. 63, No. 3, 2020

nal wall endometriosis: a surgeon's perspective and review of 445 cases. Am J Surg 2008;196:207-12.

19. Tatli F, Gozeneli O, Uyanikoglu H, Uzunkoy A, Yalcın HC, Ozgonul A, et al. The clinical characteristics and surgical approach of scar endometriosis: a case series of 14 women. Bosn J Basic Med Sci 2018;18:275-8.

20. Gidwaney R, Badler RL, Yam BL, Hines JJ, Alexeeva V, Donovan $\mathrm{V}$, et al. Endometriosis of abdominal and pelvic wall scars: multimodality imaging findings, pathologic correlation, and radiologic mimics. Radiographics 2012;32:2031-43.

21. Medeiros F, Cavalcante DI, Medeiros MA, Eleutério J Jr. Fine-needle aspiration cytology of scar endometriosis: study of seven cases and literature review. Diagn Cyto- pathol 2011;39:18-21.

22. Grigore M, Socolov D, Pavaleanu I, Scripcariu I, Grigore AM, Micu R. Abdominal wall endometriosis: an update in clinical, imagistic features, and management options. Med Ultrason 2017;19:430-7.

23. Solak A, Genç B, Yalaz S, Sahin N, Sezer TO, Solak I. Abdominal wall endometrioma: ultrasonographic features and correlation with clinical findings. Balkan Med J 2013;30:155-60.

24. Khan Z, Zanfagnin V, El-Nashar SA, Famuyide AO, Daftary GS, Hopkins MR. Risk factors, clinical presentation, and outcomes for abdominal wall endometriosis. J Minim Invasive Gynecol 2017;24:478-84. 